\title{
Radio spectral index images of the spiral galaxies NGC 0628, NGC 3627, and NGC 7331^
}

\author{
R. Paladino ${ }^{1,2}$, M. Murgia ${ }^{2,3}$, and E. Orrù ${ }^{1}$ \\ ${ }^{1}$ Institut fur Astro - und Teilchenphysik Universitat Innsbruck, Technikerstrasse 25, 6020 Innsbruck, Austria \\ e-mail: rpaladin@ca.astro.it \\ 2 INAF - Osservatorio Astronomico di Cagliari, Loc. Poggio dei Pini, Strada 54, 09012 Capoterra (CA), Italy \\ 3 Istituto di Radioastronomia - INAF, Via Gobetti 101, 40129 Bologna, Italy
}

Received 18 February 2009 / Accepted 22 May 2009

\section{ABSTRACT}

\begin{abstract}
Aims. To understand the cosmic-ray propagation mechanism in galaxies and its correlation with the sites of star formation, we compare the spatially resolved radio spectral index of three spiral galaxies with their IR distribution.

Methods. We present new low-frequency radio continuum observations of the galaxies NGC 0628, NGC 3627, and NGC 7331, taken at $327 \mathrm{MHz}$ with the Very Large Array.

Results. We complemented our data set with sensitive archival observations at $1.4 \mathrm{GHz}$ and studied the variations in the radio spectral index within the disks of these spiral galaxies. We also compared the spectral index distribution and the IR distribution, using $70 \mu \mathrm{m}$ Spitzer observations.

Conclusions. We find that in these galaxies the nonthermal spectral index is anticorrelated with the radio brightness. Bright regions, like the bar in NGC 3627 or the circumnuclear region in NGC 7331, are characterized by a flatter spectrum with respect to the underlying disk. Therefore, a systematic steepening of the spectral index with the increasing distance from the center of these galaxies is observed. Furthermore, by comparing the radio images with the $70 \mu \mathrm{m}$ images of the Spitzer satellite, we find that a similar anticorrelation exists between the radio spectral index and the infrared brightness, as expected from the local correlation between the radio continuum and the infrared emission. Our results support the idea that the electron diffusion must be efficient in regions of intense star formation. The observed anticorrelation between radio brightness and spectral index may imply that the cosmic ray density and the magnetic field strength are significantly higher in these regions than in their surroundings.
\end{abstract}

Key words. radio continuum: galaxies - galaxies: spiral - galaxies: ISM - stars: formation

\section{Introduction}

It is well known that the radio continuum of spiral galaxies consists of a mixture of nonthermal and thermal emission (e.g. Condon 1992). The nonthermal component stems from the synchrotron emission of cosmic-ray (CRe) electrons with $\mathrm{GeV}$ energies spiralling in the galaxy's magnetic field. The thermal one comes from the free-free emission from ionized gas at $T \sim$ $10^{4} \mathrm{~K}$. The two emissions are characterized by different spectra. The synchrotron component generally has a steep spectral index $\alpha \sim 0.8\left(S_{v} \propto v^{-\alpha}\right)$ and dominates at low-frequencies, while the free-free emission has a much flatter spectrum, $\alpha \simeq 0.1$, and may become relevant at frequencies greater than about $10 \mathrm{GHz}$ (Niklas \& Beck 1997). Synchrotron emission is thus best studied at frequencies below a few GHz. At these frequencies, close correlations are observed between the radio continuum and several star formation tracers like the $\mathrm{H}_{\alpha}$ emission from ionized gas, the infrared radiation (IR) from thermal dust (e.g. Yun et al. 2001), and the CO emission from molecular clouds (Rickard et al. 1977; Israel 1997; Adler et al. 1991; Murgia et al. 2002). It has been established that these correlations hold not only on global but also on local scales, down to hundreds of parsecs, within the

^ Figures 6-11 are only available in electronic form at http://www. aanda.org disks of spiral galaxies (Murgia et al. 2005; Paladino et al. 2006). Although it is commonly accepted that all these correlations ultimately originate from the process of massive stars formation itself, the details of the physical mechanisms on how this occurs are not completely understood.

One main difficulty in the study of the nonthermal radio continuum is that the synchrotron radiation essentially traces the product of CRe and magnetic field energy densities. Disentangling these two contributions is not directly possible unless making hardly verifiable assumptions (e.g. energy equipartition between field and particles). A second source of uncertainty is related to the diffusion length of CRe electrons, which is poorly known. The CRe diffuse from their injection (or reacceleration) sites and, as a consequence, their density and energy spectrum at a given location in the disk is the result of the balance between the efficiencies of different processes, such as the injection (or re-acceleration) rate, the confinement time, and the energy losses.

Since the mean free path of dust-heating photons $(\sim 100 \mathrm{pc})$ is far shorter than the presumed diffusion length of CR electrons (Bicay et al. 1989), the first attempts to model the propagation of CRe have been made by comparing radio and IR images of spiral galaxies. Bicay \& Helou (1990) smeared IRAS images of spiral galaxies by using parametrized kernels to match the 
Table 1. Summary of observations.

A. Source parameters

\begin{tabular}{ccccccc}
\hline \hline Name & $\begin{array}{c}\alpha(\mathrm{J} 2000) \\
(\mathrm{h} \mathrm{m} \mathrm{s})\end{array}$ & $\begin{array}{c}\delta(\mathrm{J} 2000) \\
\left({ }^{\circ}{ }^{\prime \prime \prime}\right)\end{array}$ & $\begin{array}{c}d \\
(\mathrm{Mpc})\end{array}$ & $\begin{array}{c}i \\
\left({ }^{\circ}\right)\end{array}$ & $\begin{array}{c}S_{\mathrm{RC}} \\
(\mathrm{mJy})\end{array}$ & $\begin{array}{c}S_{\mathrm{FIR}} \\
(\mathrm{Jy})\end{array}$ \\
\hline NGC 0628 & 013641.7 & +154659 & 7.3 & 24 & $180^{a}$ & $20.86^{c}$ \\
NGC 3627 & 112015.0 & +125930 & 11.1 & 63 & $458^{a}$ & $67.8^{d}$ \\
NGC 7331 & 223704.1 & +342456 & 15.1 & 62 & $372^{b}$ & $35.29^{c}$ \\
\hline
\end{tabular}

B. Observation details

\begin{tabular}{ccccccccc}
\hline \hline Name & $\begin{array}{c}\text { IF } \\
\text { Number }\end{array}$ & $\begin{array}{c}\text { Frequency } \\
\text { MHz }\end{array}$ & $\begin{array}{c}\text { Bandwidth } \\
\text { MHz }\end{array}$ & Configuration & $\begin{array}{c}\text { Visibility } \\
\text { Number }\end{array}$ & $\begin{array}{c}H P B W \\
" \times "\end{array}$ & $\begin{array}{c}\text { rms } \\
\text { mJy/beam }\end{array}$ & Date \\
\hline NGC 0628 & 2 & $321.5-327.5$ & 3.125 & C & 524446 & $65 \times 57$ & 2.3 & 01 Sep. 05 \\
NGC 3627 & 2 & $329.1-323.1$ & 6.250 & B & 662708 & $20 \times 16.6$ & 2 & 30 Oct. 07 \\
NGC 7331 & 2 & $329.1-323.1$ & 6.250 & B & 349475 & $17.5 \times 15.5$ & 1.1 & 03 Nov. 07 \\
\hline
\end{tabular}

A. Coordinates, distances, inclinations from Helfer et al. (2003). Inclination is defined assuming $i=0^{\circ}$ for face-on galaxies.

$S_{\mathrm{RC}}=1.4 \mathrm{GHz}$ global flux taken from (a) Condon et al. (1990) and (b) Condon (1987).

$S_{\text {FIR }}=60 \mu \mathrm{m}$ flux taken from (c) Rice et al. (1990) and from (d) the IRAS Bright Galaxy Sample (Soifer et al. 1989).

B. VLA observational properties.

observed morphology seen in the radio. They found that the diffusion length of CRe electrons is $\sim 1-2 \mathrm{kpc}$. Later, Murphy et al. (2006) applied this phenomenological image-smearing model to new Spitzer images. They found that the examined galaxies exhibit a wide range of ages and spreading scale lengths of their CR electrons, going from the youngest populations, recently injected within star-forming regions (l 500 pc), to the oldest CR electrons, making up a galaxy's underlying synchrotron disk $(l \sim 3 \mathrm{kpc})$.

The method we use to understand the cosmic ray diffusion mechanism, hence its correlation with the sites of intense star formation, is to compare spatially resolved radio spectral index images of galaxies and their IR distribution. Such a study is only now possible due to the unprecedented high spatial resolution and sensitivity of the Spitzer Space Telescope at IR wavelengths. We recently studied the point-by-point relation between the radio spectral index and the IR emission in the galaxy M 51 (Paladino et al. 2006). By comparing the Spitzer $70 \mu \mathrm{m}$ image and the spectral index image obtained between 1.4 and $4.9 \mathrm{GHz}$, we found an anticorrelation between the IR emission and the spectral index. Regions in which the IR is higher than the average tend to have a flatter radio spectrum than their surroundings. This result suggests that the CRe electrons' confinement time in the star-forming regions is shorter than their radiative lifetime and that they age as they diffuse in the disk (Murgia et al 2005).

This work would be a starting point for the program of extending this kind of analysis to a large sample of galaxies. Here we report on the spectral index analysis of three spiral galaxies: NGC 0628, NGC 3627, and NGC 7331. We observed these galaxies at $327 \mathrm{MHz}$ with the Very Large Array (VLA). To obtain detailed spectral index maps, we complemented our data set with sensitive archival observations at $1.4 \mathrm{GHz}$. Since at these frequencies the contribution from thermal emission is likely to be minimized, the spectral index can be used to study the spectrum of the nonthermal emission.

The paper is organized as follows. In Sect. 2 we describe the observations and the data reduction. The spectral index analysis and the corresponding results are reported in Sect. 3. Finally, we summarize our results in Sect. 4.

\section{Observations and data reduction}

\subsection{VLA $327 \mathrm{MHz}$ observations}

Our aim is to investigate the variations in the nonthermal spectral index in the sample of 22 nearby spiral galaxies presented in Murgia et al. (2005) and Paladino et al. (2006), by means of low-frequency radio observations. In this work we report on the first results for three galaxies taken from the afore-mentioned sample.

We present new radio observations of the spiral galaxies NGC 0628, NGC 3627, and NGC 7331 at $327 \mathrm{MHz}$ with the VLA. The basic parameters of these galaxies are listed in Table 1.A. The galaxy NGC 0628 is a clear grand-design spiral galaxy seen almost face-on. In the UV, bright knots embedded in diffuse emission trace the spiral pattern, and many of these knots are also bright in $\mathrm{H}_{\alpha}$ (Marcum et al. 2001). Futhermore, these authors suggest that the entire disk has undergone active star formation within the past $500 \mathrm{Myr}$ and that the inner regions have experienced more rapidly declining star formation than the outer regions. NGC 3627, the brightest member of the Leo Triplet, is an interacting spiral galaxy with a bar and two asymmetric spiral arms. The western arm is accompanied by weak traces of star formation, while the eastern arm contains a straight actively starforming segment in its inner part (Soida et al. 2001). NGC 7331 is a relatively highly inclined spiral galaxy with a ring, visible in CO (e.g. Regan et al. 2001), radio continnum (e.g. Cowan et al. 1994), submillimeter (Bianchi et al. 1998), and Spitzer-infrared (Regan et al. 2004; Regan et al. 2006) images.

We observed NGC 3627 and NGC 7331 with the VLA in B configuration, while NGC 0628 was observed with the VLA in C configuration. A summary of the observations, including the VLA configuration, frequency, bandwidth, visibilities number, date of observations, is reported in Table 1.B.

The observations were conducted in spectral line mode (see Table 1.B for details on frequencies and bandwidths), in order to reduce the effects of bandwidth smearing and to allow for more accurate radio-frequency interference (RFI) excision.

The data were calibrated and imaged with the NRAO package AIPS (Astronomical Image Processing System). Both flux 


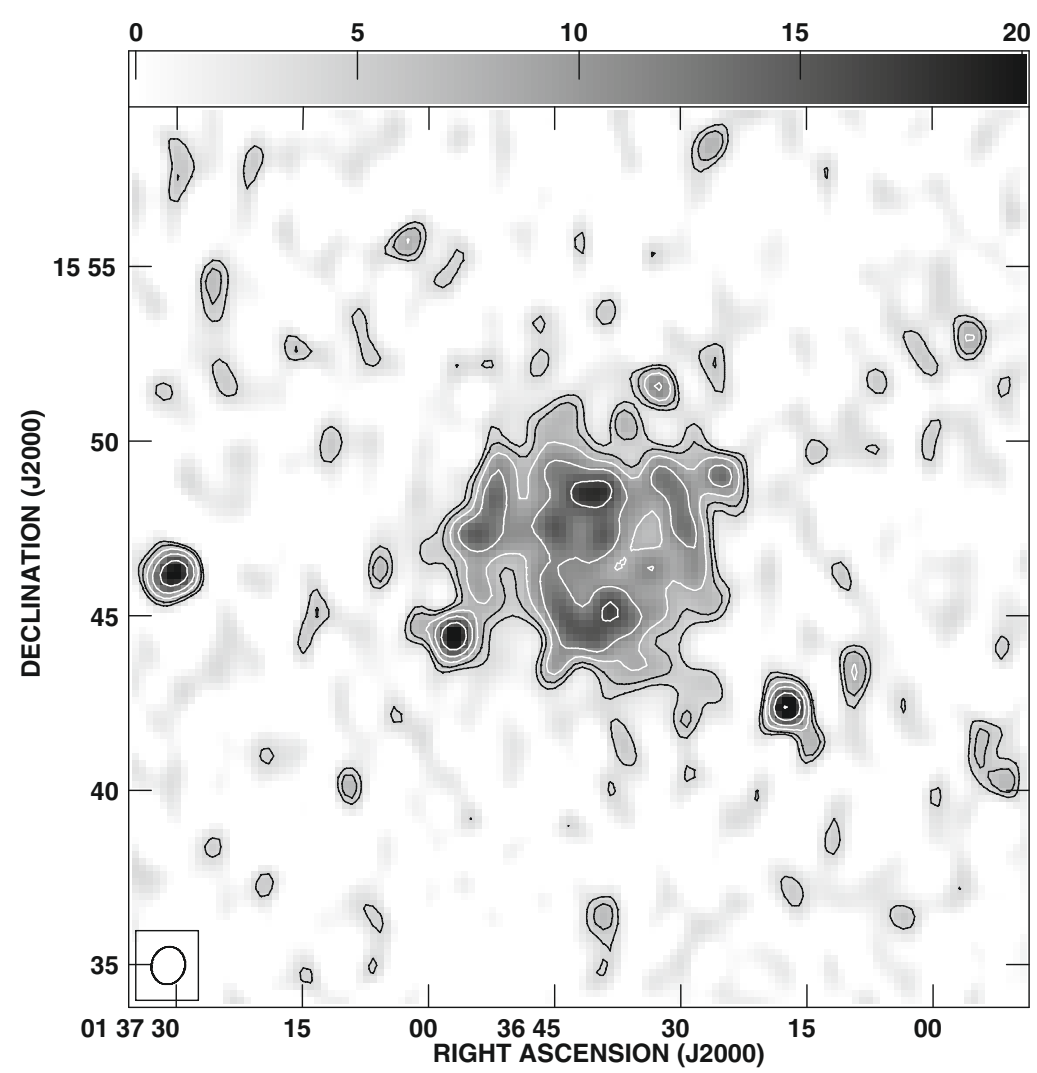

Fig. 1. Galaxy NGC 0628: radio image at $327 \mathrm{MHz}$. The $F W H M$ is $65.4^{\prime \prime} \times 57^{\prime \prime}$. Contour levels start from a level of $2 \sigma$ (i.e. $4 \mathrm{mJy} / \mathrm{beam}$ ) and scale by a factor of $\sqrt{2}$. Grey scale flux range is $0-20 \mathrm{mJy} /$ beam.

density and bandpass calibration were based directly on observations of the strong calibrator sources 3C 48, 3C 123, 3C 147, and $3 \mathrm{C} 286$. The amplitude gains were tied to the flux scale of Baars et al. (1977). For the initial phase calibration we used further calibrators closest to the observed sources, when available. A careful data editing operation was made to remove RFI present in individual channels. At the end of this procedure, $\sim 10 \%$ of data were flagged in the observations of NGC 0628. A higher fraction of data, about $35 \%$ and $28 \%$ for NGC 3627 and NGC 7331, respectively, was flagged in the 2007 runs, when some EVLA antennas started to be included in the VLA.

The visibility data were spectrally averaged, retaining 5 channels with a resolution of $488 \mathrm{KHz}$ for NGC 0628, and 6 channels with a resolution of $781 \mathrm{KHz}$ for NGC 3627 and NGC 7331.

The entire field was imaged using the AIPS program IMAGR in $3 \mathrm{D}$ mode. Owing to its large field of view at $327 \mathrm{MHz}$, the VLA is not coplanar at this frequency. Therefore, the primary beam was divided into small overlapping "facets" over which the VLA can be safely assumed to be coplanar (Cornwell \& Perley 1992). Confusion lobes of surrounding sources far from the center of the field are often so strong that their sidelobes contribute measurably to the noise level in the central region. Thus, by using the NRAO VLA Sky Survey (NVSS) catalog as a reference, we also cleaned the strongest sources over an area of about $\sim 60^{\circ}$ in radius by placing small facets around each of them (task SETFC).

Final images were created by an iterative process of CLEANing and self-calibration including all these "facets". In the self-calibration process, we used only the strongest components inside the primary beam in order to increase the accuracy of the phase calibration model. The use of smaller clean boxes decreased the possibility of "overcleaning" and minimized the CLEAN bias (see Condon et al. 1998a, for details). Figures 1-3 show the $327 \mathrm{MHz}$ images of the three observed sources, with typical noise level reported in Table 1.B. NGC 0628, which has the largest angular extent $\left(\sim 6^{\prime}\right)$ among the three galaxies, has been observed with the VLA in C configuration with an angular resolution of $65^{\prime \prime} \times 57^{\prime \prime}$. The observations of NGC 3627 and NGC $7331\left(\sim 5^{\prime}\right)$ were taken with the VLA in B configuration at a resolution of $20^{\prime \prime} \times 16.6^{\prime \prime}$ and $17.5^{\prime \prime} \times 15.5^{\prime \prime}$, respectively.

\subsection{Archival VLA observations at $1.4 \mathrm{GHz}$}

For the purposes of the spectral index imaging, we complemented our data set with archival observations at $1.4 \mathrm{GHz}$ with the VLA in D and C configurations. The archival data we recovered is described in Table 2. The data were calibrated and imaged with the package AIPS following the standard procedures. The coverage of the spatial frequencies of the $1.4 \mathrm{GHz}$ observations is very similar to that at $327 \mathrm{MHz}$. However, to ensure a perfect match in angular resolution, we convolved the final images at both frequencies to the largest beam (see Sect. 3.2).

\subsection{IR Spitzer images}

The galaxies studied in this work were observed as part of the Spitzer infrared nearby galaxies survey (SINGS, Kennicutt et al. 2003) and IR images have been released. For details on observations and data processing, see delivery document for Fifth (and last) SINGS Data Delivery, April $2007^{1}$.

\footnotetext{
1 http://data.spitzer. caltech. edu/popular/ sings/20070410_enhanced_v1/Documents/ sings_fifth_delivery_v2.pdf
} 


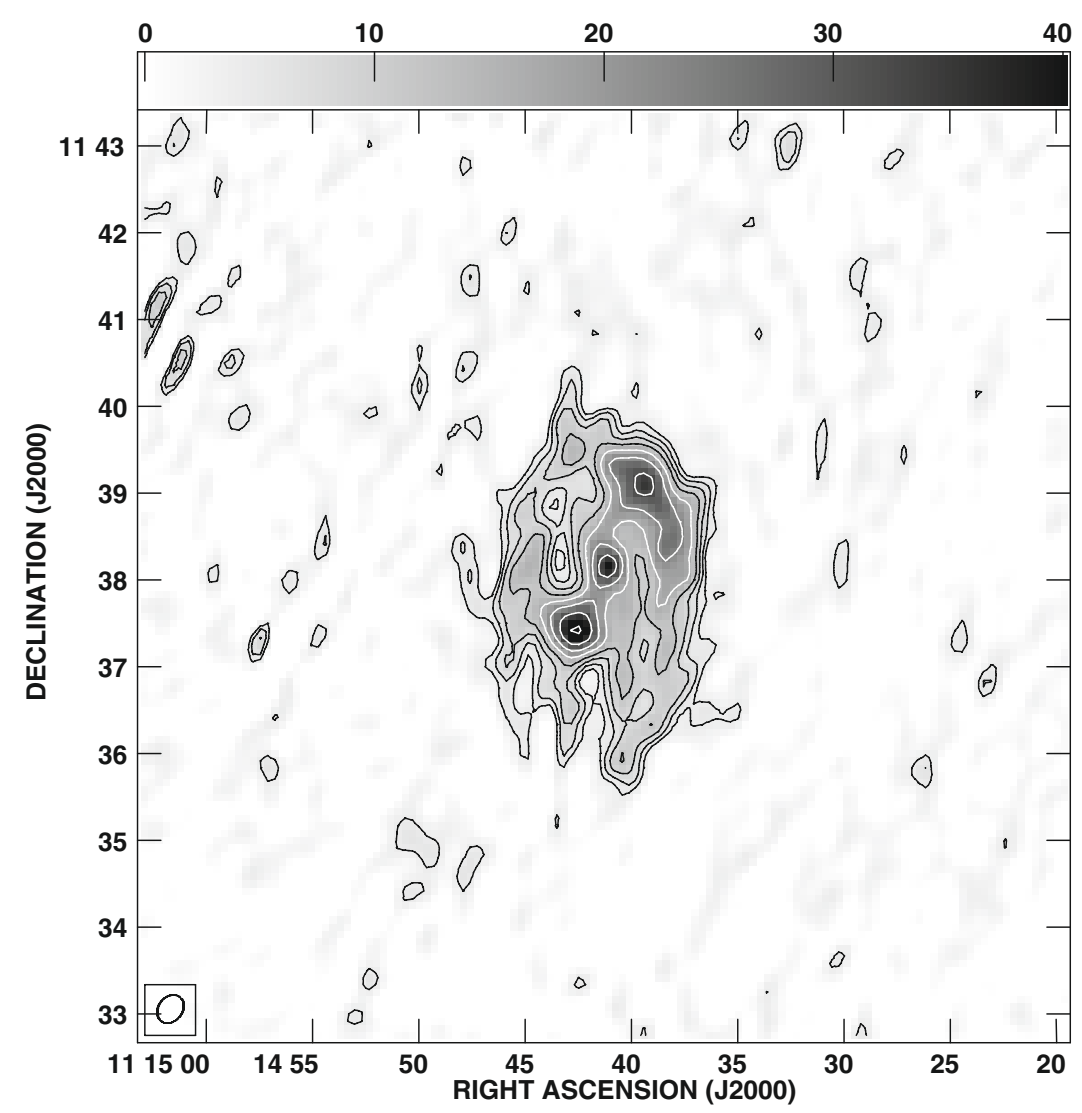

Fig. 2. Galaxy NGC 3627: radio image at $327 \mathrm{MHz}$. The $F W H M$ is $21.0^{\prime \prime} \times 16.6^{\prime \prime}$. Contour levels start from a level of $2 \sigma$ (i.e. $4 \mathrm{mJy} / \mathrm{beam}$ ) and scale by a factor of $\sqrt{2}$. Grey scale flux range is $0-40 \mathrm{mJy} / \mathrm{beam}$.

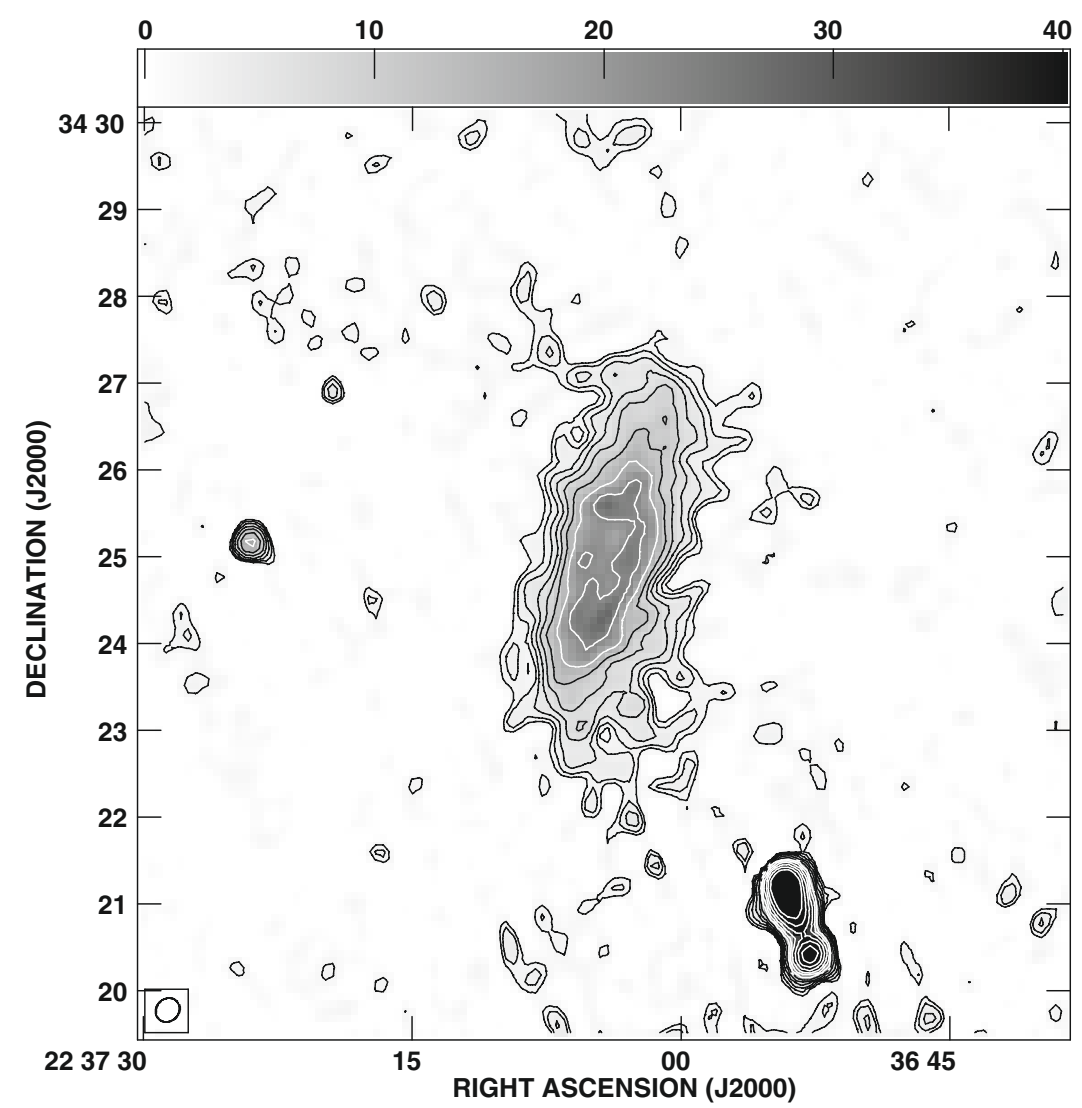

Fig. 3. Galaxy NGC 7331: radio image at $327 \mathrm{MHz}$. The $F W H M$ is $17.5^{\prime \prime} \times 15.5^{\prime \prime}$. Contour levels start from a level of $2 \sigma$ (i.e. $2 \mathrm{mJy} / \mathrm{beam}$ ) and scale by a factor of $\sqrt{2}$. Grey scale flux range is $0-40 \mathrm{mJy} / \mathrm{beam}$. 
Table 2. VLA archival observations at $1.4 \mathrm{GHz}$.

\begin{tabular}{ccccc}
\hline \hline Source & $\begin{array}{c}\text { VLA } \\
\text { config }\end{array}$ & $\begin{array}{c}H P B W \\
" \times "\end{array}$ & $\begin{array}{c}\text { rms } \\
\mu \mathrm{Jy} / \text { beam }\end{array}$ & Program ID \\
\hline NGC 0628 & $\mathrm{D}$ & $42.72 \times 40.06$ & 54 & AA161 \\
NGC 3627 & $\mathrm{C}$ & $16.52 \times 14.02$ & 150 & AS541 \\
NGC 7331 & $\mathrm{C}$ & $17.49 \times 15.48$ & 40 & AB345 \\
\hline
\end{tabular}

The observations obtained with the Multiband Imaging Photometer for Spitzer (MIPS, Rieke et al. 2004) at $70 \mu \mathrm{m}$ have resolution of $18^{\prime \prime}$. We convolved these images to the beam of our $327 \mathrm{MHz}$ radio images in order to compare the spectral index distribution with the IR one. As reported in the data release, due to special processing, the quality of the NGC $733170 \mu \mathrm{m}$ data is somewhat worse than the quality of the other $70 \mu \mathrm{m} \mathrm{im}-$ ages released. However, we use this image to compare the spectral index and IR distribution in NGC 7331, handling the results obtained from this analysis with care.

\section{Analysis and results}

\subsection{Integrated radio spectra}

We measured total flux densities at $327 \mathrm{MHz}$ from the new images of the observed galaxies. The values obtained are reported in Table 3.

To investigate whether some absorption process affects the emission from these galaxies, in particular in the range of frequencies between $327 \mathrm{MHz}$ and $1.4 \mathrm{GHz}$, we analyzed their integrated radio spectra. From a careful assessment of published values between 57 and $5000 \mathrm{MHz}$, we derived the integrated radio spectra of the three observed galaxies. The values of the integrated flux density are reported in Table 3. The telescopes from which the observations were taken are reported along with each reference.

Most of the measurements were tied to the flux scale of Baars et al. (1977). Some values (reported with an indication in Table 3) are on the scale of Wyllie (1969), which Baars et al. (1977) concluded is very close to their own.

Figure 4 shows the integrated radio spectra of NGC 0628, NGC 3627, and NGC 7331, in which our new $327 \mathrm{MHz}$ flux density measurements are indicated by filled triangles. The integrated spectrum of NGC 0628 is shown in the left panel of Fig. 4. The data are very well-fitted by a single power law with a spectral index of $\alpha=0.78$. This value is not unusual for a spiral galaxy; the average spectral index of spiral galaxies is $0.74 \pm 0.03$ (Gioia et al. 1982).

The integrated spectra of galaxies NGC 3627 and NGC 7331 present deviations from the classical power law. Most spiral galaxies do indeed show a change in spectral index with frequency and different possible reasons have been proposed (e.g., Israel \& Mahoney 1990; Hummel 1991; Pohl \& Schlickeiser 1992). We fitted the integrated spectra of these galaxies by introducing an absorption at low frequency. This seems to be necessary in particular to fit the low frequency point in NGC 7331. The integrated spectra were fitted with a power law, $S_{\text {Pow }}(v) \propto v^{-\alpha}$, modified by low-frequency absorption as in Pacholczyk (1970):

$S(v) \propto\left(v / v_{\text {peak }}\right)^{(\alpha+2.5)}\left(1-\mathrm{e}^{-\left(v / v_{\text {peak }}\right)^{-(\alpha+2.5)}}\right) \cdot S_{\text {Pow }}(v)$

where $v_{\text {peak }}$ is the frequency at which the optical depth is equal to 1 , while $\alpha$ is the spectral index in the optically thin power-law
Table 3. Integrated flux densities on the observed sources.

\begin{tabular}{|c|c|c|c|}
\hline Source & $\begin{array}{c}\text { Frequency } \\
\mathrm{MHz}\end{array}$ & $\begin{array}{c}\text { Flux density } \\
\text { Jy }\end{array}$ & Reference \\
\hline \multirow{5}{*}{ NGC 0628} & 57.5 & $2 \pm 1$ & 1 \\
\hline & 324.4 & $0.49 \pm 0.03$ & This work \\
\hline & 1400 & $0.15 \pm 0.01$ & 2 \\
\hline & 1515 & $0.16 \pm 0.01$ & This work \\
\hline & 4850 & $0.060 \pm 0.005$ & 3 \\
\hline \multirow[t]{9}{*}{ NGC 3627} & 57.5 & $2.3 \pm 0.7$ & 1 \\
\hline & 160 & $1.6 \pm 0.3^{a}$ & 4 \\
\hline & 325.5 & $0.97 \pm 0.03$ & This work \\
\hline & 408 & $0.93 \pm 0.05^{a}$ & 5 \\
\hline & 611 & $0.87 \pm 0.1^{a}$ & 4 \\
\hline & 1370 & $0.5 \pm 0.01$ & 6 \\
\hline & 1425 & $0.38 \pm 0.01$ & This work \\
\hline & 2695 & $0.2 \pm 0.04$ & 4 \\
\hline & 4850 & $0.14 \pm 0.02$ & 7 \\
\hline \multirow[t]{8}{*}{ NGC 7331} & 74 & $1.12 \pm 0.18$ & 8 \\
\hline & 325 & $0.935 \pm 0.004$ & 9 \\
\hline & 325.9 & $0.94 \pm 0.02$ & This work \\
\hline & 1489 & $0.32 \pm 0.01$ & This work \\
\hline & 1400 & $0.33 \pm 0.01$ & 2 \\
\hline & 1369 & $0.35 \pm 0.01$ & 6 \\
\hline & 2380 & $0.18 \pm 0.01$ & 10 \\
\hline & 4850 & $0.096 \pm 0.013$ & 7 \\
\hline
\end{tabular}

${ }^{a}$ Flux density scale from Wyllie (1969).

References and telescopes: 1. Israel \& Mahoney (1990): Clark Lake radio Telescope; 2. Condon et al. (1998b): NVSS - NRAO VLA D array; 3. Gregory et al. (1996): NRAO Green Bank Telescope; 4. Haynes et al. (1975): CSIRO telescope; 5. Large et al. (1981): Molonglo Radio Telescope; 6. Braun et al. (2007): Westerbork Telescope WSRT; 7. Gregory \& Condon (1991): NRAO Green Bank; 8. Cohen et al. (2007): NRAO VLA B array; 9. Rengelink et al. (1997): WENSS WSRT; 10. Dressel \& Condon (1978): Arecibo Telescope.

regime. For the optically thick regime, we assumed a spectral index 2.5 , as expected in the case of an homogeneous synchrotron self-absorbed source. The fitted parameters, along with the reduced $\chi^{2}$ values $\left(\chi^{2} / \mathrm{ndf}\right.$, where ndf is the number of degrees of freedom), are reported in Table 4 . The frequency peaks for both galaxies resulting from the fit are at $v_{\text {peak }} \lesssim 100 \mathrm{MHz}$, thus the region between $327 \mathrm{MHz}$ and $1.4 \mathrm{GHz}$, should be relatively free of absorption.

A hint of steepening is observed in the spectra above a frequency of $\sim 1 \mathrm{GHz}$. We tried to fit the data with the continuous injection model (CI) described in Murgia et al. (1999). In this model it is assumed that the radio sources are continuously replenished by constant flow of fresh relativistic particles with a power-law energy distribution $\left(N(E) \propto E^{-\delta}\right)$ and that the magnetic field is constant, the radio spectrum has a standard shape (Kardashev 1962), with injection spectral index $\alpha_{\text {inj }}=(\delta-1) / 2$ below a critical frequency $\nu_{\mathrm{br}}$, and $\alpha=\alpha_{\mathrm{inj}}+0.5$ above $\nu_{\mathrm{br}}$. The steepening of the spectral index at high frequencies stems from particle energy losses.

By comparing the $\chi^{2} /$ ndf values, we can notice that the observed high-frequency spectra are better fitted by a CI model, rather than a power law, for both galaxies. In NGC 3627 the CI model fit does not require synchrotron self-absorption. Nevertheless, the interpretation of the high frequency break 

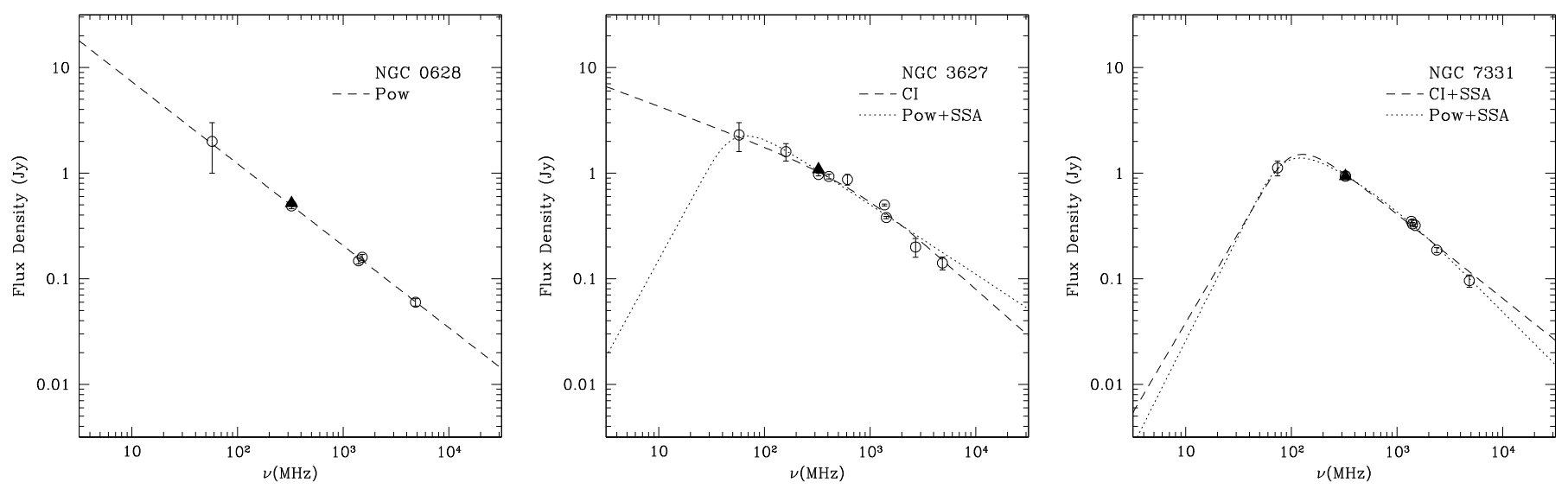

Fig. 4. Spectral energy distribution of the observed spiral galaxies.

Table 4. Results of the fit with models in detail in Sect. 3.1.

\begin{tabular}{lccccc}
\hline \hline Source & Model & \multicolumn{4}{c}{ fitted parameters } \\
& & $\alpha$ & $v_{\text {br }}$ & $v_{\text {peak }}$ & $\chi^{2} /$ ndf \\
& & & $\mathrm{MHz}$ & $\mathrm{MHz}$ & \\
\hline NGC 0628 & Pow & 0.78 & - & - & 0.76 \\
\hline NGC 3627 & Pow+SSA & 0.66 & - & 54 & 4.3 \\
& CI & 0.4 & 937 & - & 2.9 \\
\hline NGC 7331 & Pow+SSA & 0.79 & - & 112 & 2.5 \\
& CI+SSA & 0.5 & 1025 & 92 & 0.98 \\
\hline
\end{tabular}

Pow $=$ Power law. SSA $=$ Synchrotron self absorption. $\mathrm{CI}=$ Continuous injection.

should be considered carefully as the assumptions of the CI model too simple to describe the complex structures and physics of spiral galaxies.

In closing, the analysis of integrated spectra of the three galaxies considered in this work shows that the region between $327 \mathrm{MHz}$ and $1.4 \mathrm{GHz}$ should be relatively free of absorptions.

\subsection{Local variations in the radio spectral index}

We investigate the variations in the nonthermal spectral index as a function of position within the galaxies. The integrated spectra show that the region between $327 \mathrm{MHz}$ and $1.4 \mathrm{GHz}$ is relatively free of (self-)absorption, even in the case of NGC 7331 where a low-frequency turnover seems to be present. Moreover, at these frequencies the contribution from thermal emission is likely to be minimized, and thus spectral index can be used to study the spectrum of the nonthermal emission.

The spectral index images between $327 \mathrm{MHz}$ and $1.4 \mathrm{GHz}$, were obtained by considering only those pixels where the brightness was above $3 \sigma$ at both frequencies. To accurately determine the spectral index distribution identical beam size and shape at different frequencies is required. We convolved NGC $0628 \mathrm{im}-$ ages to a 66" round beam, while for NGC 3627 and NGC 7331 we used a beam of $20^{\prime \prime}$. In addition, to avoid any positional offsets, the images were aligned and interpolated to identical projections and field center. The primary beam correction was applied to the images at both frequencies.

The spectral index images, calculated between $327 \mathrm{MHz}$ and $1.4 \mathrm{GHz}$, of the three sources are shown in left hand panels of Fig. 6, 8, and 10. The dynamic range of images at $327 \mathrm{MHz}$ is typically lower than the one at $1.4 \mathrm{GHz}$, therefore the cut in these spectral index maps in most of the points is driven by the $327 \mathrm{MHz}$ images. Right panel of the same figures show the spectral index uncertainty. The comparison of the $\alpha$ image with the error image show that most of the observed spectral index features are statistically significant.

The $66^{\prime \prime}$ beam of NGC 0628 partly hidden the spiral structure of the galaxy, bright clumps in spiral arms in radio images correspond to flatter regions in the $\alpha$ image (Fig. 6). The spectral index image of NGC 3627 (Fig. 8) clearly shows that the bar and its bright ending regions have a flatter spectral index than the underlying disk. The radio spectrum of NGC 7331 (Fig. 10) is almost uniformly flat in the galaxy's center and steepens towards the peripheral regions of the disk.

For a quantitative analysis we measured the point-to-point brightness on the radio images at $327 \mathrm{MHz}$ and $1.4 \mathrm{GHz}$, as follows. We overlaid regular grids of rectangular beam-sized boxes on the radio images, and we averaged the brightness in each box. We then calculated the spectral index between $327 \mathrm{MHz}$ and $1.4 \mathrm{GHz}$.

Figures 5, 7, 9, and 11 show the results of this point-to-point comparison. Only points exceeding 5 times the rms of the computed spectral index are shown. This severe clipping increases our confidence in spectral index variations.

Figure 5 shows the variations in spectral index with the radio brightness in these three galaxies. A common feature in these galaxies is that the spectral index is anticorrelated with the radio brightness. Bright regions, such as the bar in NGC 3627 or the circumnuclear region in NGC 7331, are characterized by a flatter spectrum than the underlying disk. In all three galaxies, the brighter regions have a spectral index of about $\alpha \simeq 0.5-0.6$. In the faintest regions, the radio spectrum steepens to $\alpha \simeq 1.0-1.2$.

The left hand panels of Figs. 7, 9, and 11 show the spectral index versus the distance from the center of the sources. The anticorrelation between the brightness and the spectral index causes a systematic steepening of the radio spectrum with the increasing distance from the center of these galaxies, where typically brightest regions are located.

\subsection{Comparison with IR emission}

The far infrared luminosity from galaxies is emitted by interstellar dust heated by the general interstellar radiation field and the more intense radiation in star formation regions. The $70 \mu \mathrm{m}$ emission can be used as a star formation diagnostic. To investigate the connection between star formation sites and cosmic rays 

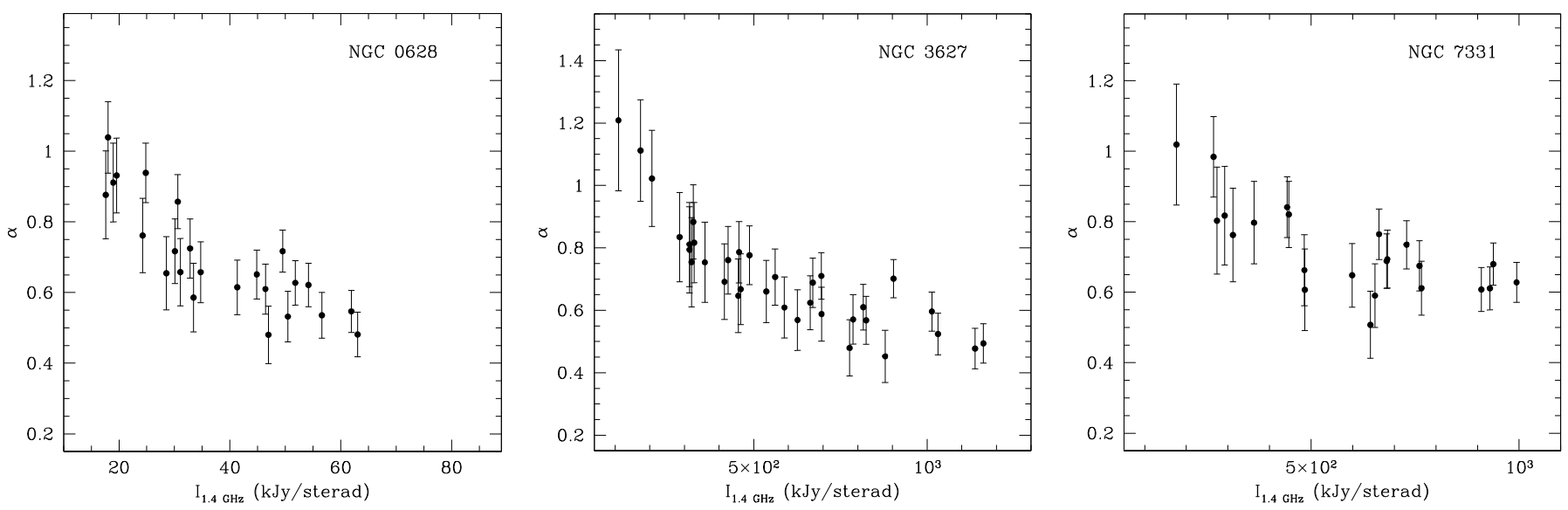

Fig. 5. Radio spectral index variation with $1.4 \mathrm{GHz}$ brightness for the three studied galaxies.

propagation mechanism, we compared $70 \mu \mathrm{m}$ distribution to the spectral index in galaxies.

We used $70 \mu \mathrm{m}$ Spitzer images (Kennicutt et al. 2003) convolved to the same beam of our spectral images. We measured the IR brightness using the same grid as described in Sect. 3.2. Right hand panels in Figures 7, 9, and 11 show the radio spectral index versus the $70 \mu \mathrm{m}$ brightness. Despite the very different morphologies, the radio spectral index shows a common behavior in all three galaxies: an anticorrelation exists between the radio spectral index and the infrared brightness. Regions in which the IR is higher than the average tend to have flatter radio spectra than their surroundings. The radio spectral index shows the same anticorrelation as the radio brightness. The observed trend is expected from the local correlation between the radio continuum and the infrared emission. This result is consistent with the idea that the CRe electrons' confinement time in the star-forming regions is shorter than their radiative lifetime.

\section{Summary}

We have presented new $327 \mathrm{MHz}$ VLA radio images of the three spiral galaxies NGC 0628, NGC 3627, and NGC 7331. Based on these images, we measured the integrated flux densities and performed a spatially resolved study of the synchrotron spectral index distribution.

We calculated the integrated radio spectra of these galaxies, using the measured integrated fluxes, together with flux densities measurements taken from the literature. In the considered frequency range, the radio spectrum of NGC 0628 is well-fitted by a single power law with an index $\alpha \simeq 0.79$. The integrated spectra of galaxies NGC 3627 and NGC 7331 present deviations from the classical power law. We fitted these integrated spectra and noticed that the observed high-frequency spectra are reproduced better by a continuous injection model. In NGC7331 a synchrotron self-absorption process reproduces the low-frequency spectrum. This is one of the possible mechanisms producing the low-frequency turnover and more low-frequency observations are needed to confirm it.

We complemented our data set with sensitive archival observations at $1.4 \mathrm{GHz}$ and studied the variation in the radio spectral index to within the disks of these spiral galaxies. We find that the spectral index is anticorrelated with the radio brightness. Bright regions, like the bar in NGC 3627 or the circumnuclear region in NGC 7331, are characterized by a flatter spectrum with respect to the underlying disk. This causes a systematic steepening of the spectral index with the increasing distance from the center of these galaxies. Furthermore, by comparing the radio images with the $70 \mu \mathrm{m}$ images of the Spitzer satellite, we find that a similar anticorrelation exists between the radio spectral index and the infrared brightness, as expected from the local correlation between the radio continuum and the infrared emission. Our results support the idea that in regions of intense star formation, where the injection rate of electrons is presumably higher, the CRe electrons' confinement times are shorter than their radiative lifetimes (Murgia et al. 2005), so the electron diffusion must be efficient. We also observed the anticorrelation between RC brightness and spectral index, which may imply that the cosmic ray density and the magnetic field strength are significantly higher in these regions than in their surroundings.

Finally, we note that this study adds to a growing list of examples confirming the importance of high-resolution, lowfrequency radio observations in providing an important tool for understanding the physical mechanism of production and diffusion of cosmic rays. This also points to the strong potential for the new generation of much more sensitive low-frequency instruments, such as LOFAR. Future work will involve a detailed comparison of the radio spectral index distribution and the starformation tracers, such as IR and molecular emission, in a larger sample of spiral galaxies, in order to analyze the connection of cosmic ray production and propagation with the star formation processes.

Acknowledgements. We would like to thank the anonymous referee for insightful comments that improved this manuscript. E.O. acknowledges financial support by the Austrian Science Foundation (FWF) through grant number P18523N16. The National Radio Astronomy Observatory is operated by Associated Universities, Inc., under contract to the National Science Fundation. This research made use of the NASA/IPAC Extragalactic Database (NED), which is operated by the Jet Propulsion Laboratory, California Institute of Technology, under contract with the National Aeronautics and Space Administration and of CATS database Astrophysical CATalogs support System.

\section{References}

Adler, D. S., Allen, R. J., \& Lo, K. Y. 1991, ApJ, 382, 475

Baars, J. W. M., Genzel, R., Pauliny-Toth, I. I. K., \& Witzel, A. 1977, A\&A, 61, 99

Bianchi, S., Alton, P. B., Davies, J. I., \& Trewhella, M. 1998, MNRAS, 298, L49 Bicay, M. D., \& Helou, G. 1990, ApJ, 362, 59

Bicay, M. D., Helou, G., \& Condon, J. J. 1989, ApJ, 338, L53

Braun, R., Oosterloo, T. A., Morganti, R., Klein, U., \& Beck, R. 2007, A\&A, 461,455

Cohen, A. S., Lane, W. M., Cotton, W. D., et al. 2007, AJ, 134, 1245 
Condon, J. J. 1987, ApJS, 65, 485

Condon, J. J. 1992, ARA\&A, 30, 575

Condon, J. J., Helou, G., Sanders, D. B., \& Soifer, B. T. 1990, ApJS, 73, 359

Condon, J. J., Cotton, W. D., Greisen, E. W., et al. 1998a, AJ, 115, 1693

Condon, J. J., Cotton, W. D., Greisen, E. W., et al. 1998b, AJ, 115, 1693

Cornwell, T. J., \& Perley, R. A. 1992, A\&A, 261, 353

Cowan, J. J., Romanishin, W., \& Branch, D. 1994, ApJ, 436, L139

Dressel, L. L., \& Condon, J. J. 1978, ApJS, 36, 53

Gioia, I. M., Gregorini, L., \& Klein, U. 1982, A\&A, 116, 164

Gregory, P. C., \& Condon, J. J. 1991, ApJS, 75, 1011

Gregory, P. C., Scott, W. K., Douglas, K., \& Condon, J. J. 1996, ApJS, 103, 427

Haynes, R. F., Huchtmeier, W. K. G., \& Siegman, B. C. 1975, A compendium of radio measurements of bright galaxies, Melbourne: Commonwealth Scientific and Industrial Research Organization (CSIRO), Division of Radiophysics

Helfer, T. T., Thornley, M. D., Regan, M. W., et al. 2003, ApJS, 145, 259

Hummel, E. 1991, A\&A, 251, 442

Israel, F. P. 1997, A\&A, 328, 471

Israel, F. P., \& Mahoney, M. J. 1990, ApJ, 352, 30

Kardashev, N. S. 1962, Soviet Astronomy, 6, 317

Kennicutt, Jr., R. C., Armus, L., Bendo, G., et al. 2003, PASP, 115, 928

Large, M. I., Mills, B. Y., Little, A. G., Crawford, D. F., \& Sutton, J. M. 1981, MNRAS, 194, 693

Marcum, P. M., O'Connell, R. W., Fanelli, M. N., et al. 2001, ApJS, 132, 129
Murgia, M., Fanti, C., Fanti, R., et al. 1999, A\&A, 345, 769

Murgia, M., Crapsi, A., Moscadelli, L., \& Gregorini, L. 2002, A\&A, 385, 412

Murgia, M., Helfer, T. T., Ekers, R., et al. 2005, A\&A, 437, 389

Murphy, E. J., Helou, G., Braun, R., et al. 2006, ApJ, 651, L111

Niklas, S., \& Beck, R. 1997, A\&A, 320, 54

Pacholczyk, A. G. 1970, Radio astrophysics. Nonthermal processes in galactic and extragalactic sources, Series of Books in Astronomy and Astrophysics (San Francisco: Freeman)

Paladino, R., Murgia, M., Helfer, T. T., et al. 2006, A\&A, 456, 847

Pohl, M., \& Schlickeiser, R. 1992, A\&A, 263, 37

Regan, M. W., Thornley, M. D., Helfer, T. T., et al. 2001, ApJ, 561, 218

Regan, M. W., Thornley, M. D., Bendo, G. J., et al. 2004, ApJS, 154, 204

Regan, M. W., Thornley, M. D., Vogel, S. N., et al. 2006, ApJ, 652, 1112

Rengelink, R. B., Tang, Y., de Bruyn, A. G., et al. 1997, A\&AS, 124, 259

Rice, W., Boulanger, F., Viallefond, F., Soifer, B. T., \& Freedman, W. L. 1990, ApJ, 358, 418

Rickard, L. J., Turner, B. E., \& Palmer, P. 1977, ApJ, 218, L51

Rieke, G. H., Young, E. T., Engelbracht, C. W., et al. 2004, ApJS, 154, 25

Soida, M., Urbanik, M., Beck, R., Wielebinski, R., \& Balkowski, C. 2001, A\&A, 378,40

Soifer, B. T., Boehmer, L., Neugebauer, G., \& Sanders, D. B. 1989, AJ, 98, 766 Wyllie, D. V. 1969, MNRAS, 142, 229

Yun, M. S., Reddy, N. A., \& Condon, J. J. 2001, ApJ, 554, 803 
R. Paladino et al.: Radio spectral index images of the spiral galaxies NGC 0628, NGC 3627, and NGC 7331, Online Material p 1
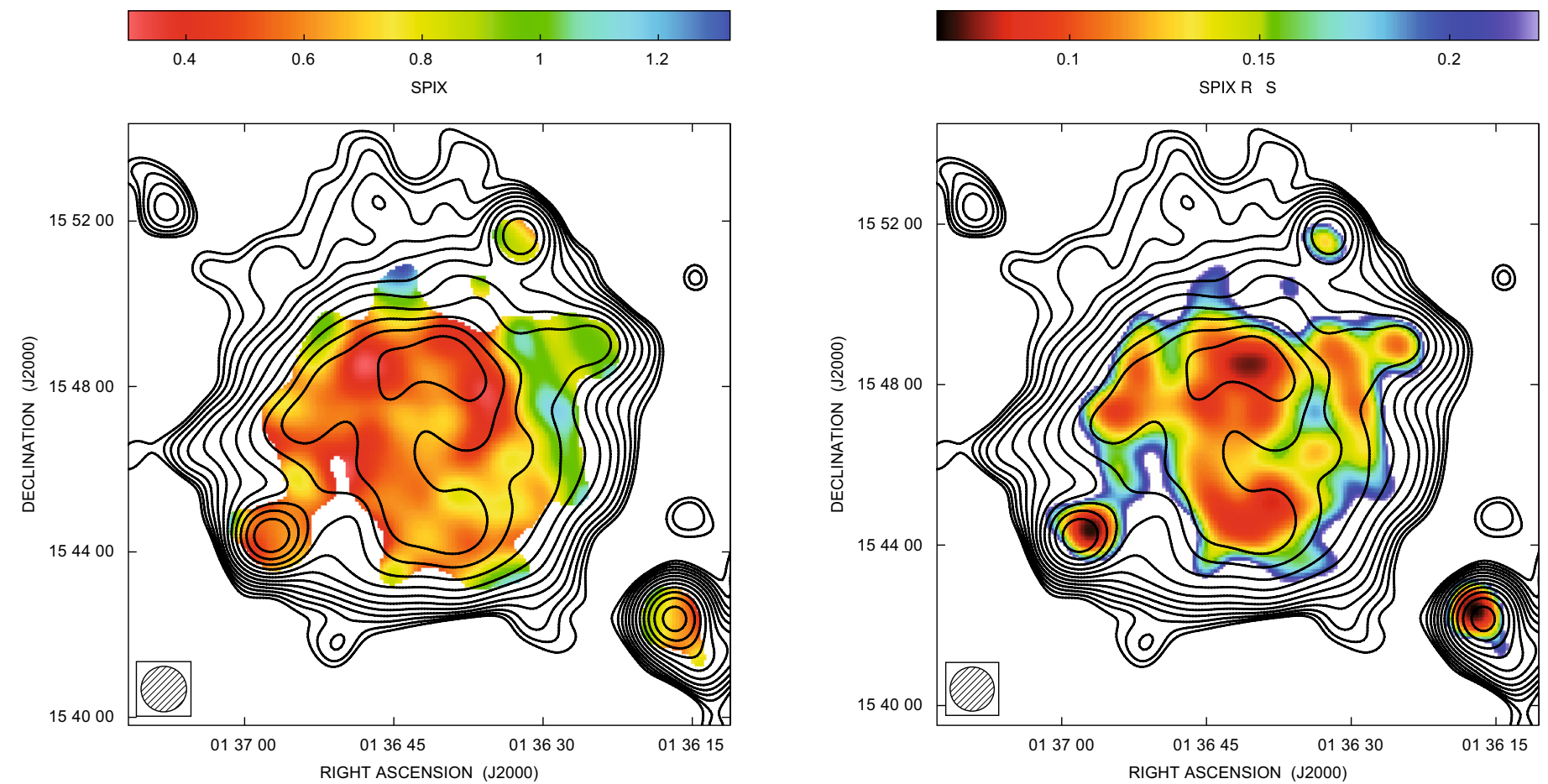

Fig. 6. Galaxy NGC 0628 - Left panel: the color scale represents the image of the spectral index measured between $327 \mathrm{MHz}$ and $1.4 \mathrm{GHz}$. Pixels with an intensity level above $3 \sigma$ at both frequencies have been considered. Contours levels of the radio image at $1.4 \mathrm{GHz}$, starting from $3 \sigma$ and scaling by a factor of $\sqrt{2}$, are overlaid on the image. The FWHM of this image is $66^{\prime \prime} \times 66^{\prime \prime}$. Right panel: the color scale represents the image of the spectral index uncertainty.
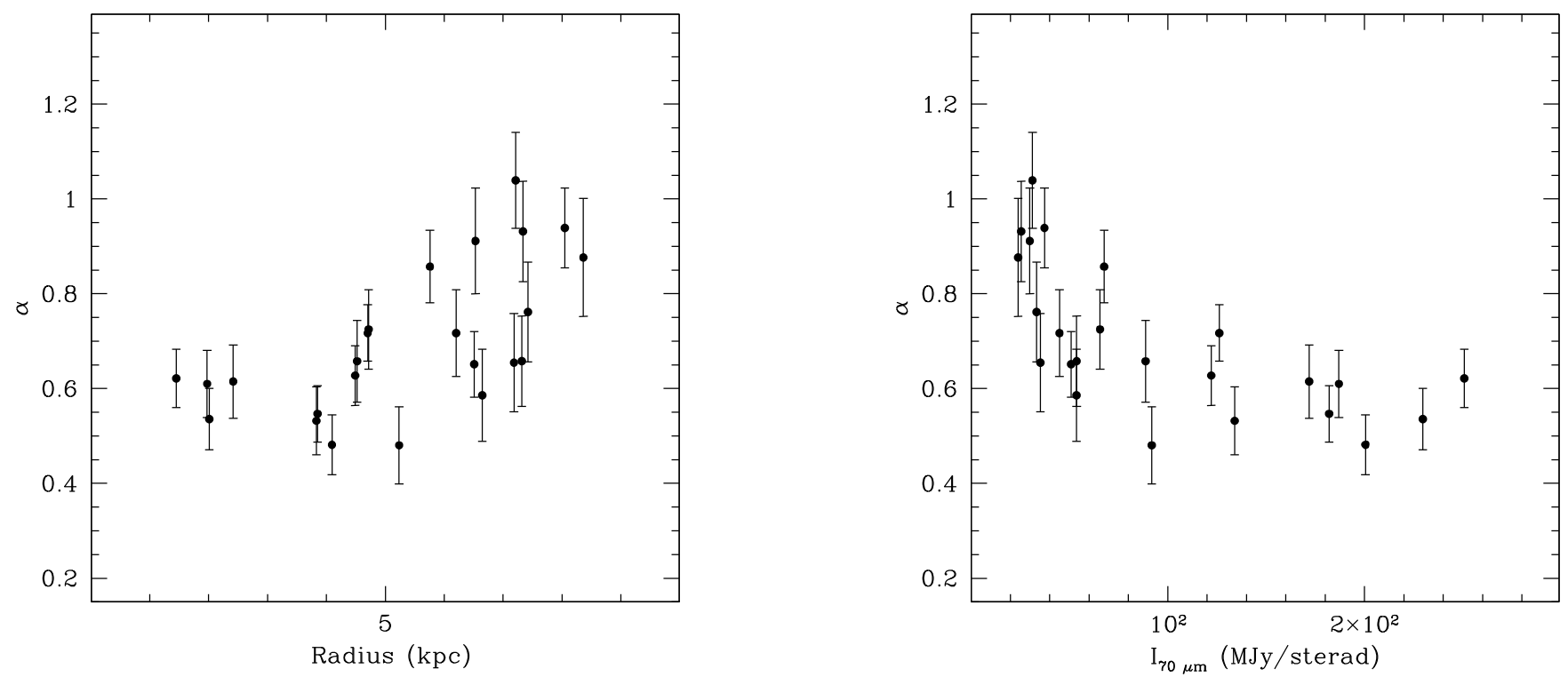

Fig. 7. Galaxy NGC 0628: spectral index radial profile (left panel) and spectral index variation with IR-70 $\mu$ m brightness (right panel). 
R. Paladino et al.: Radio spectral index images of the spiral galaxies NGC 0628, NGC 3627, and NGC 7331, Online Material p 2
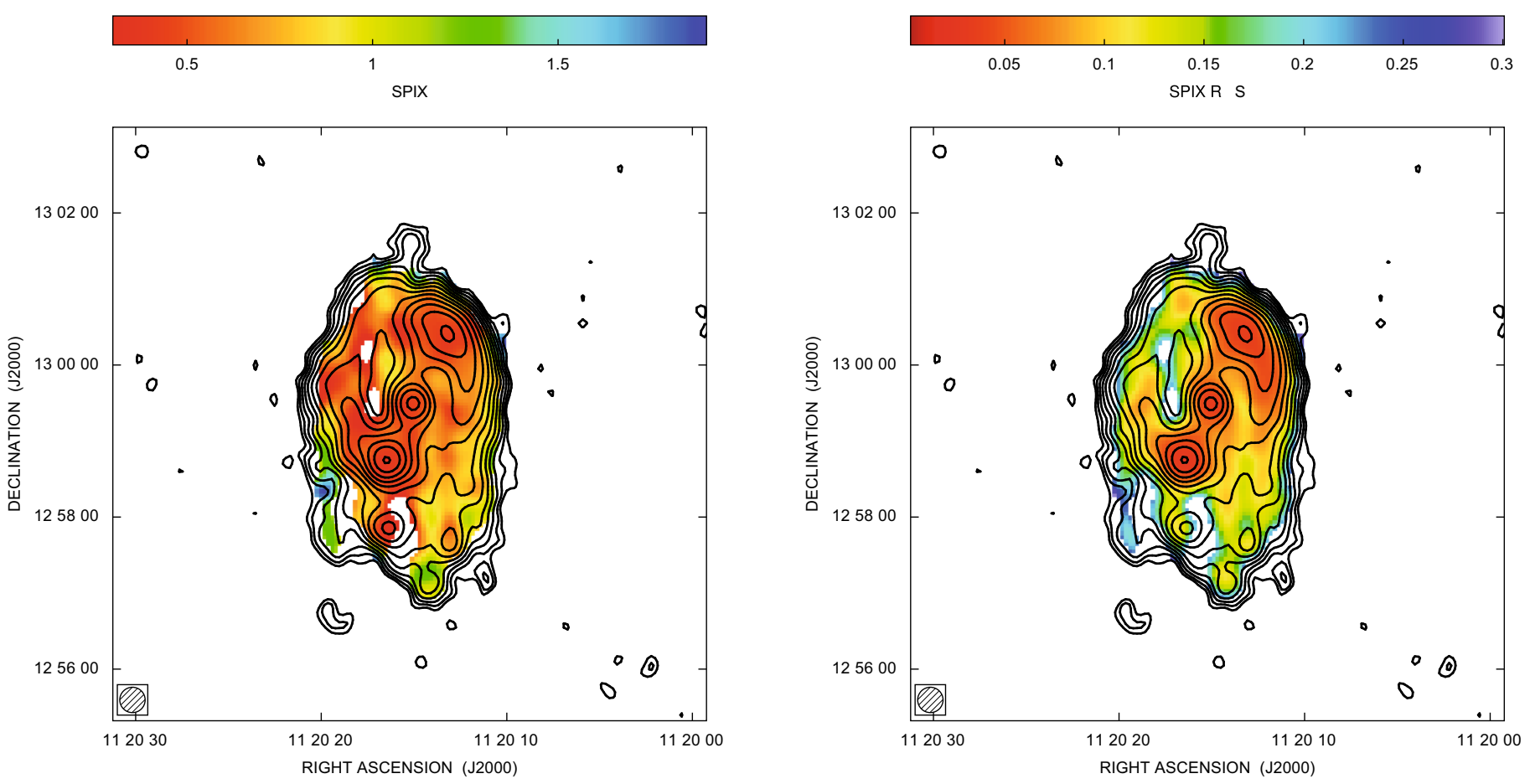

Fig. 8. Galaxy NGC 3627 - Left panel: the color scale represents the image of the spectral index measured between $327 \mathrm{MHz}$ and $1.4 \mathrm{GHz}$. Pixels with an intensity level above $3 \sigma$ at both frequencies have been considered. Contours levels of the radio image at $1.4 \mathrm{GHz}$, starting from $3 \sigma$ and scaling by a factor of $\sqrt{2}$, are overlaid on the image. The $F W H M$ of this image is $20^{\prime \prime} \times 20^{\prime \prime}$. Right panel: the color scale represents the image of the spectral index uncertainty.
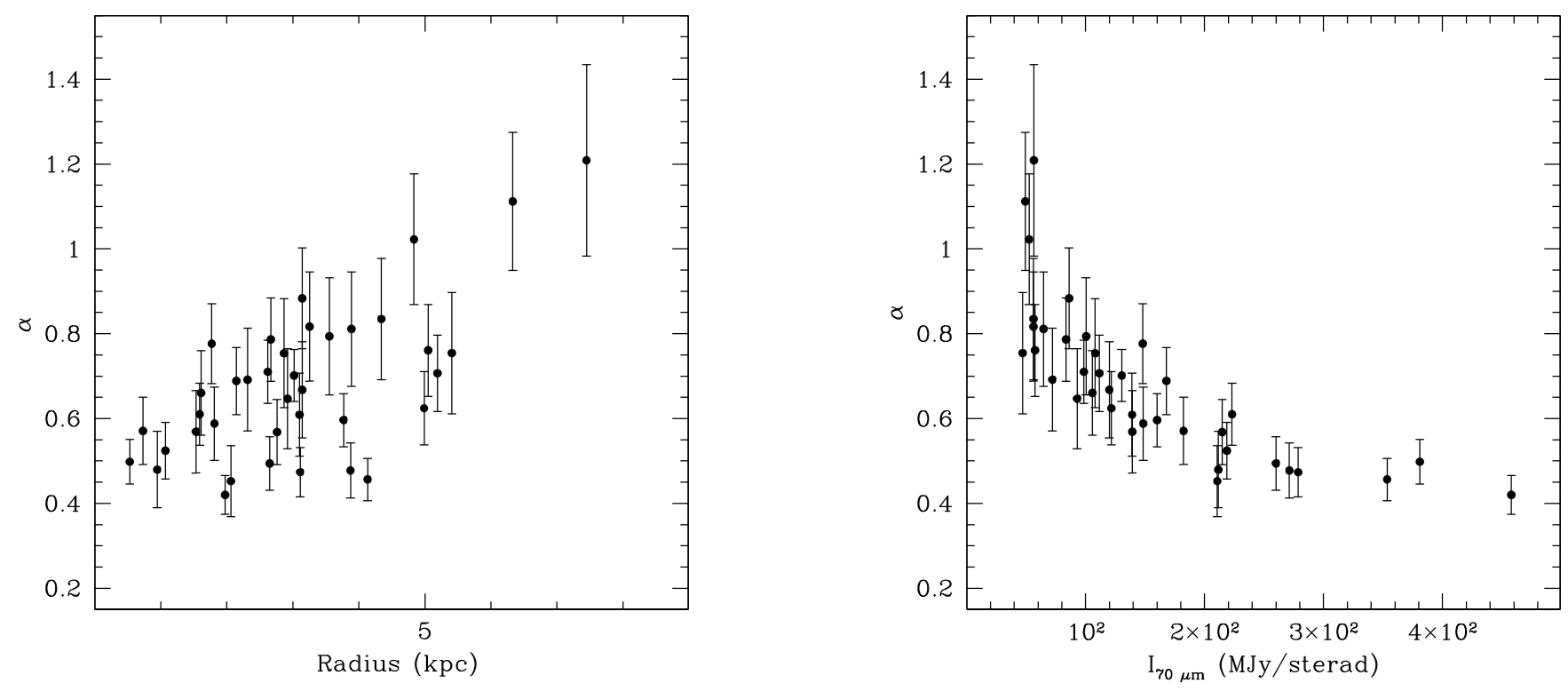

Fig. 9. Galaxy NGC 3627: Spectral index radial profile (left panel) and spectral index variation with IR-70 $\mu$ m brightness (right panel). 
R. Paladino et al.: Radio spectral index images of the spiral galaxies NGC 0628, NGC 3627, and NGC 7331, Online Material p 3
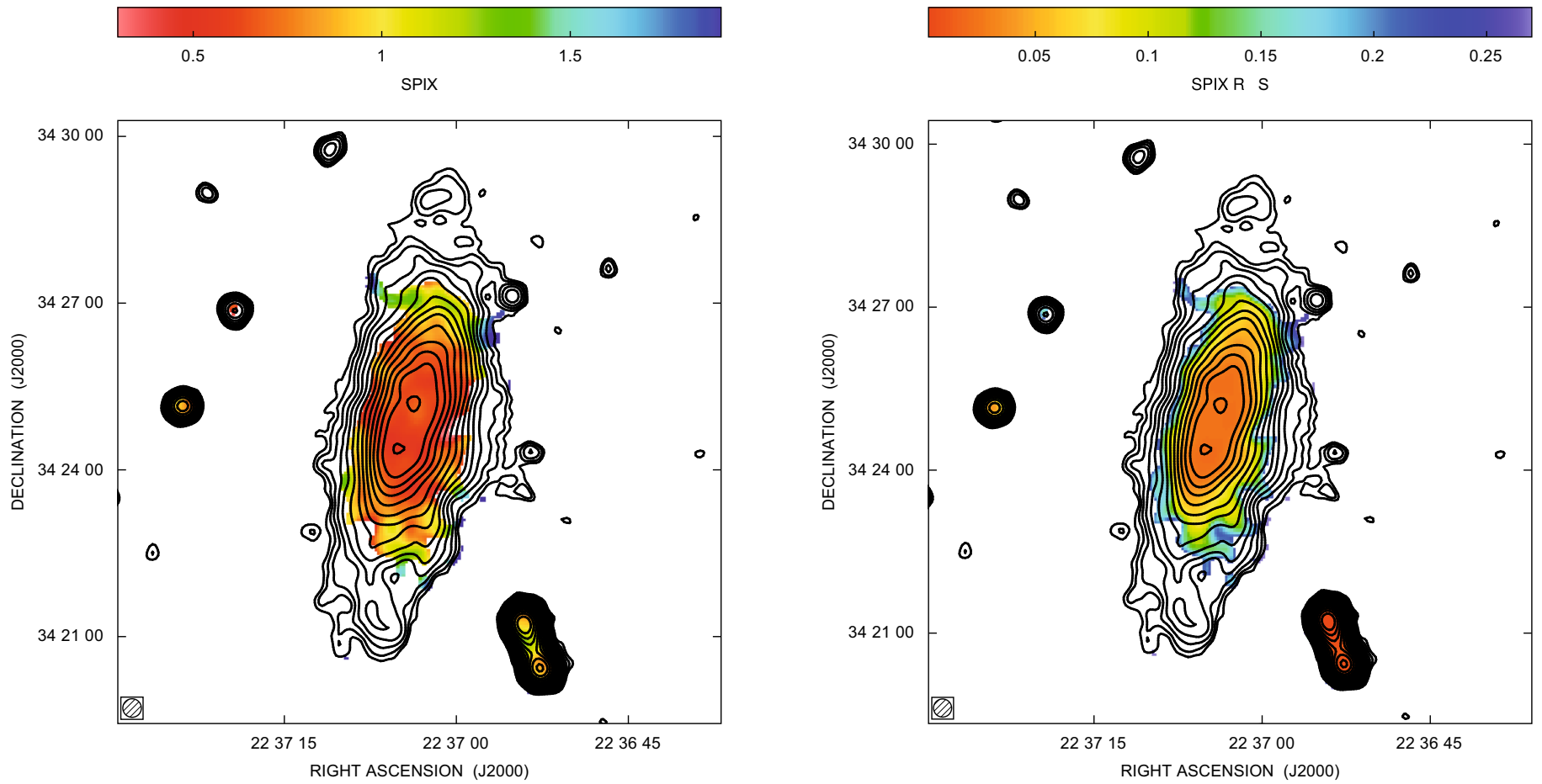

Fig. 10. Galaxy NGC 7331 - Left panel: the color scale represents the image of the spectral index measured between $327 \mathrm{MHz}$ and $1.4 \mathrm{GHz}$. Pixels with an intensity level above $3 \sigma$ at both frequencies have been considered. Contours levels of the radio image at $1.4 \mathrm{GHz}$, starting from $3 \sigma$ and scaling by a factor of $\sqrt{2}$, are overlaid on the image. The FWHM of this image is $20^{\prime \prime} \times 20^{\prime \prime}$. Right panel: the color scale represents the image of the spectral index uncertainty.
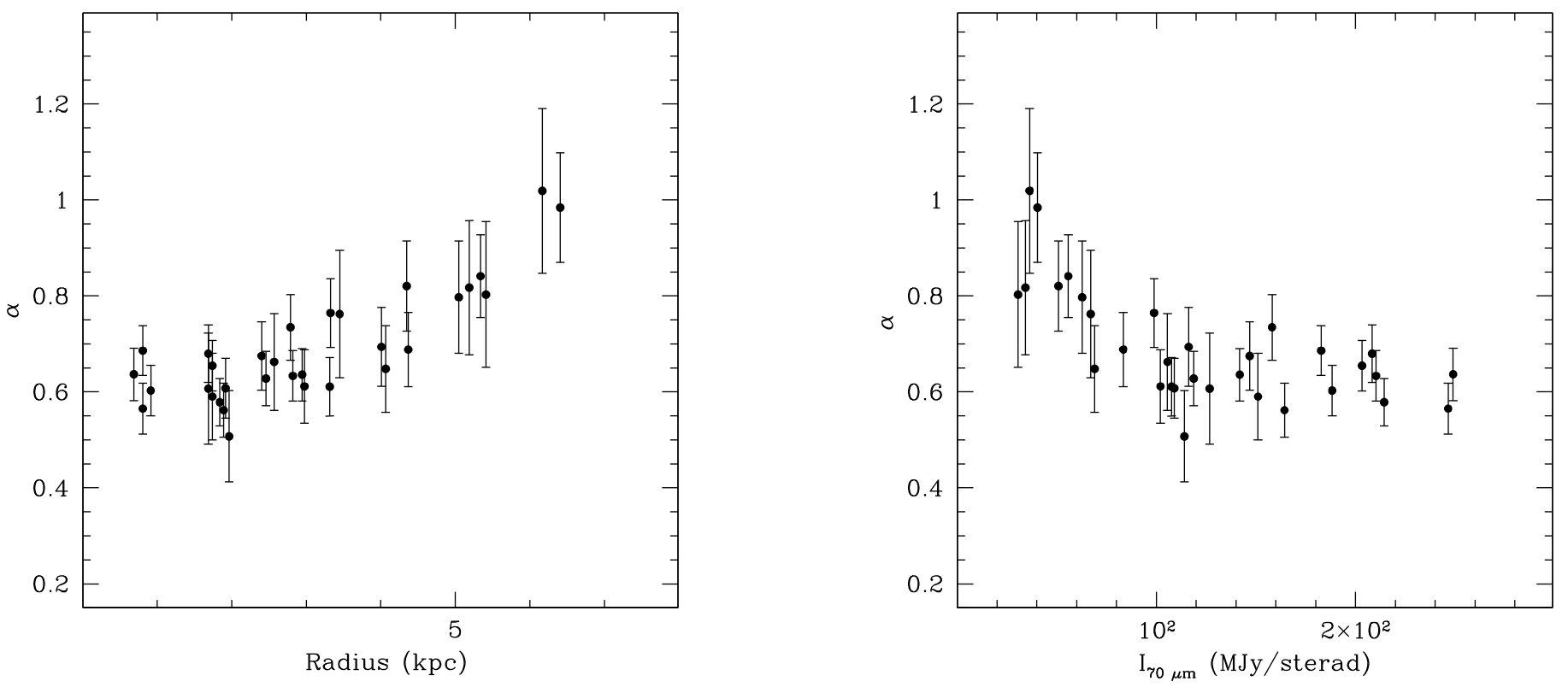

Fig. 11. Galaxy NGC 7331: spectral index radial profile (left panel) and spectral index variation with IR-70 $\mu$ m brightness (right panel). 